\title{
La educación como derecho: desde la inclusión para la diversidad y a lo largo de la vida
}

\author{
Education as a right: from inclusion to diversity and throughout life
}

\author{
Ana Cristina Quesada Monge ${ }^{1}$
}

Fecha de recepción: 25-02-2020

Fecha de aceptación: 1-10-2020

\begin{abstract}
Resumen
El presente artículo representa un análisis bibliográfico de lo que es y significa la educación como un derecho, donde su reflexión se realiza desde dos ejes o propuestas educativas: la inclusión para la diversidad y a lo largo de la vida. Estos dos ejes son considerados, por el Marco de Acción de la Educación hacia el 2030 (Declaración de Incheon), como elementos fundamentales para generar un cambio paradigmático en las concepciones y prácticas en la educación, a fin de alcanzar una sociedad más justa y equitativa. Mediante la revisión bibliográfica de artículos científicos en las bases de datos de textos completos del Sistema de Bibliotecas de la Universidad de Costa Rica, EBSCO y Google Educativo, durante los años 2000 hasta 2020, fue posible dar respuesta a cuatro preguntas generadoras, planteadas por la autora, para elaborar el presente escrito, bajo la modalidad de ensayo. Fue así como se elaboró un breve recorrido conceptual de la educación como derecho, desde las normativas internacionales, para luego conocer las propuestas de cambio que fomentan los dos ejes de interés del estudio. Luego se rescatan dos discusiones y preocupaciones que autores latinoamericanos plantean sobre los temas de análisis, para finalmente exponer algunos retos y desafíos en su aplicabilidad, desde la mirada de la autora, en el contexto contemporáneo.
\end{abstract}

Palabras claves: Educación como derecho, educación inclusiva para la diversidad, a largo de la vida, retos y desafíos.

\begin{abstract}
This document represents a bibliographic analysis about the education and its means as a human right, furthermore, the analysis is carried out from two educational axes or proposals: inclusion for diversity and throughout life. These two axes are considered, by the Education Action Framework towards 2030 (Incheon Declaration), as main elements to generate a paradigmatic change in the conceptions and practices in the education, in order to achieve a more just and equitable society.

Through the bibliographic review of scientific articles in the full-text databases of the Library System of the University of Costa Rica, EBSCO and Google Educativo, during the years 2000 to 2020, it was possible to answer four general questions, which were exposed the author, to elaborate the present document, under the modality of test. This is how a brief conceptual tour of education as a human right was developed, based on international regulations, to later learn about the proposals for change that promote the two axes of study interest. Then, two discussions and concerns that Latin American authors raise about the analysis topics are rescued, to finally expose some challenges and challenges in their applicability, from the author's point of view, in the contemporary context.
\end{abstract}

Keywords: Education, education inclusive for diversity, lifelong education, challenges and obstacles.

\footnotetext{
1 Máster en Trabajo Social, con énfasis en Gerencia Social. Estudiante del doctorado en Educación, Universidad Estatal a Distancia. Docente de la Carrera de Trabajo Social, Sede de Occidente, Universidad de Costa Rica, Costa Rica. Correo electrónico: cristina.quesada@ucr.ac.cr
} 


\section{Introducción}

La educación como derecho es un tema que históricamente aparece en diferentes textos científicos; no obstante, su análisis y discusión tienen un matiz particular en la coyuntura de los últimos veinte años, producto de los cambios y transformaciones vividos por la humanidad en el ámbito político, económico, social y cultural. Una de estas particularidades es la integración de dos ejes de trabajo en este campo: la educación inclusiva para la diversidad y a lo largo de la vida. Se plantearon cuatro preguntas generadoras que motivaron el acercamiento y análisis bibliográfico de los temas en cuestión y permitieron elaborar el presente artículo científico, bajo la modalidad de ensayo: cómo se ha posicionado la educación como derecho, cuál es la especificidad de la educación desde la inclusión para la diversidad y a lo largo de la vida, cuáles son las principales discusiones y preocupaciones de estos temas en el contexto latinoamericano y cuáles son los principales retos y desafíos para su aplicabilidad en el contexto contemporáneo.

La revisión bibliográfica se realizó sobre artículos científicos que aparecieron bajo los términos de "educación como derecho", "educación inclusiva” y "educación a lo largo de la vida", en las bases de datos de textos completos del Sistema de Bibliotecas de la Universidad de Costa Rica, el EBSCO y Google Académico durante los años 2000 hasta 2020. Mediante las técnicas de análisis de contenido, fue posible identificar información como conceptos, hechos históricos, datos referenciales, reflexiones de los investigadores, entre otros más, que al agruparlos en categorías de análisis, permitieron dar respuesta a las preguntas orientadoras del presente documento. Por consiguiente, este artículo se estructura en tres apartados, así como las conclusiones.

El primero se enfoca en reconstruir conceptualmente la educación como derecho, desde los aportes de las normativas internacionales, que han reconocido y legitimado mundialmente este derecho, para luego centrarse en conocer la propuesta actual de educación inclusiva para la diversidad y a lo largo de la vida. Como segundo apartado, se exponen dos discusiones o preocupaciones que autores latinoamericanos refieren de este tema analizado en el continente. Finalmente, se identifican algunos retos y desafíos por resolver para su aplicabilidad en el contexto contemporáneo, así como las conclusiones del estudio.

\section{La evolución conceptual de la educación como un derecho: desde la inclusión para la diversidad y a lo largo de la vida}

El reconocimiento internacional de la educación como un derecho tiene sus bases del Pensamiento Republicano Cívico. Para la investigadora Calderón (2015, p. 4), la propuesta de la educación en el Estado Moderno se nutre desde este pensamiento, el cual tiene sus cimientos de la Teoría Política del Republicanismo y se nutre de la tradición griega y romana, especialmente de los planteamientos de Aristóteles y Cicerón, al concebir que el ser humano tiene una virtud civil o cívica. Uno de los postulados del pensamiento republicano, según esta investigadora, es fomentar prácticas democráticas e igualitarias entre los seres humanos y las comunidades, es decir, un estilo de vida cívico, como garante para alcanzar los principios de justicia, paz, armonía, comprensión y tolerancia. Adicionalmente, para la misma investigadora, la propuesta de la educación está condicionada al régimen político de cada Estado Parte, donde lamentablemente, algunos países de corte autoritario o totalitario tienden a condicionar o violentar el alcance de dicho derecho. Es por eso que organismos internacionales, tales como las Naciones Unidas y la UNESCO, entre otros, han visto la necesidad de crear instrumentos jurídicos internacionales (tratados), que reconocen de manera global (homogénea y obligatoria) este papel y funciones esenciales de la educación en el ser humano y en la sociedad.

El primer instrumento jurídico internacional que reconoció la educación como derecho fue la Declaración Universal de los Derechos Humanos (1948), el cual expuso que toda persona por su sola condición humana tiene un conjunto de derechos fundamentales, entre ellos la educación, los cuales son inviolables. Posteriormente, con el Pacto Internacional de los Derechos Económicos, Sociales y Culturales (1966) lo visualizó para aquellas personas que tienen una condición de discriminación y desventaja social. Con la Declaración Mundial de la 
Educación para Todos (1990), se amplía nuevamente este concepto, al reconocer la condición de universalidad del alcance y disfrute de la educación, sin importar la condición etaria, género, etnia, credo religioso, entre otro (Zurita, 2011, Calderón, 2015 y Ruiz, 2020).

El conjunto de estas normativas internacionales consideran que la educación es una condición intrínseca e irrenunciable de todo ser humano, es decir, que toda persona, sin importar su condición económica, social, cultural, étnica, género, entre otras, tiene la garantía de su disfrute en cualquier contexto mundial.

El fin último de la educación es fomentar el desarrollo integral del ser humano en la sociedad y a la vez fortalecer el disfrute y alcance de otros derechos y libertades fundamentales. Por otro lado, se establece que la sociedad, mediante el papel del Estado, debe garantizar las condiciones para que todas las personas tengan acceso a la educación básica y de manera gratuita (Zurita, 2011 y Calderón, 2015). En tal sentido, con las anteriores intenciones, la educación cumple una función que trasciende el medio de instrucción o capacitación de las condiciones básicas del ser humano en la sociedad; pretende ser un:

“amplio espectro de experiencias vitales y de procesos de aprendizaje que permitan al ser humano, ya sea de manera individual o colectiva, desarrollar su personalidad, dotes y aptitudes y llevar una vida plena y satisfactoria en el seno de la sociedad" (Naciones Unidas, 2001, p.2).

La anterior idea es importante porque la educación se convierte en un medio y factor protector del desarrollo integral del ser humano en la sociedad. Para alcanzar esta condición protectora, las mismas normativas reconocen cuatro principios que deben garantizar el disfrute de la educación: disponibilidad (debe haber instituciones y programas de enseñanza en cantidad suficiente para que todas las personas tengan acceso a ellas), accesibilidad (la no discriminación, accesibilidad económica y material de las personas o colectivos para el disfrute de este derecho), aceptabilidad (los programas y métodos pedagógicos deben ser aceptables para los estudiantes, sus padres o tutores) y la adaptabilidad (la educación ha de tener la flexibilidad necesaria para que se adapte a las necesidades de sociedades y comunidades en transformación, para responder a las necesidades de los alumnos en contextos culturales y sociales) (Naciones Unidas, 1999, p.3).

Es así como los instrumentos jurídicos internacionales promueven y fomentan políticas y lineamientos estatales, para expandir la educación en todos los niveles y a todos los grupos poblacionales. Lo anterior ha generado el fenómeno denominado por algunos investigadores como la "ampliación en la cobertura de los servicios educativos", la cual se manifestó con gran fuerza a partir de los años 90, en todos los niveles educativos, con especial énfasis en los niveles de la primaria y secundaria. Con esta intención y esfuerzo de expandir e integrar el derecho a la educación en todos los sectores y grupos poblacionales, se manifiestan ciertas situaciones de disparidad y desigualdad, que se convierten en elementos violadores y discriminatorios de este derecho (Gluz, Andrade y Rodríguez, 2018 y Castillo, 2019), porque existe un interés por integrar al ser humano al sistema educativo, desde la visión homogénea, estandarizada y única, que no se adecua a todas las diferentes manifestaciones del ser humano y la sociedad.

Por otra parte, investigadores como Goutier (2012, citado por Crespo, 2018) y Gluz et al., 2018), consideran que las políticas educativas de corte neoliberal, que surgen a inicio del XXI, tienen otro rumbo diferente, que afecta el espíritu de fomentar la educación como derecho, pues poseen el matiz de fomentar lineamientos políticos compensatorios, asistenciales y focalizadores, especialmente para las poblaciones en condición de pobreza o desventaja social.

Esta intención se percibe con los Objetivos del Desarrollo del Milenio 2000, dado que visualiza la educación para aliviar las situaciones de disparidad social, entre ellos la pobreza (Zurita, 2011). Es aquí donde se fomentan reformas educativas, que buscan combatir la pobreza, mediante los programas y servicios educativos. Este último planteamiento es fuertemente criticado por sectores y estudiosos de la educación como derecho, dado que visualiza una forma de minimizar o reducir el valor y aporte de la educación para el ser humano y la sociedad. 
Por ejemplo, Torres (2005) expone que el hecho de concebir la educación como un alivio a la pobreza ha sido una de las principales debilidades, dado que le resta el sentido de verla como fundamento del desarrollo y empoderamiento del ser humano en la sociedad. Por su parte, Goutier (2012) expresa que la educación es vista como un "hecho económico", con una pérdida de sentido, porque se limita a concebirla desde una ideología pragmática, es decir, solo busca resultados medibles y respuesta rápidas (citado por Crespo, 2018, p. 39). Por consiguiente, estos investigadores recomiendan que uno de los principales retos y desafíos de la educación en el presente siglo XXI es recuperar su sentido como un derecho, más que verla como un medio de enfrentar las condiciones o contradicciones generados por el sistema capitalista.

Se observa un esfuerzo de la Declaración de Incheon (La Educación, F. 2015), la cual se presenta como el Marco de Acción de la Educación hacia el 2030, para rescatar este ámbito de desarrollo como un derecho inherente del ser humano. Un aspecto que llama la atención en este instrumento internacional es que incorpora dos ejes, que resultan tener una visión novedosa en el alcance y práctica de la educación como derecho, los cuales se presentan seguidamente.

\subsection{Educación inclusiva para la diversidad}

La educación inclusiva ha tenido una evolución paradigmática a lo largo de la historia. En sus inicios, la atención de la diversidad fue vista desde un modelo médico asistencial, cuya finalidad fue "normalizar" a las personas. Posteriormente, surgen modelos como la educación especial o integradora, que si bien representan un inmenso avance en la atención a la diversidad, lo hacen desde un paradigma del déficit, es decir, responsabiliza la deficiencia en el individuo y no en la sociedad. Existen grandes críticas generadas por movimientos sociales alrededor del tema, por ejemplo los que abordan el sector de la discapacidad, que identifican que dicha propuesta no genera un cambio en los contextos causantes de las situaciones de exclusión y discriminación del ser humano. Por eso, estos grupos demandaron cambiar esta mirada con un enfoque más inclusivo. Es así como las tendencias internacionales y nacionales, a principios del presente siglo, buscan que la diversidad sea atendida desde el modelo inclusivo, caracterizado por posicionar las dificultades en los procesos de enseñanza y aprendizaje y no en la forma de aprendizaje del ser humano.

Se distinguen entonces dos paradigmas de concepción y atención de la diversidad: uno desde el déficit, en el cual las diferencias son concebidas como una enfermedad o una deficiencia de las personas; y el otro, que es llamado paradigma inclusivo, donde las diferencias se interpretan como un valor y un derecho que debe ser considerado por el contexto para ofrecer posibilidades de desarrollo (López, 2003 citado por Amaro, 2018, p. 54). No obstante, en la última década, se está integrando otro componente distintivo al paradigma de la educación inclusiva, el cual es reconocer la diversidad. La diversidad está dada porque todas las personas tienen rasgos que las unen y las identifican como grupos sociales, pero a la vez cada una es diferente, por lo que este constructo implica un elemento de identidad humana. Para Parrilla (1999), la diversidad no es solo una cuestión meramente conceptual, sino que es una manera de pensar, hacer y actuar consigo mismo y la sociedad (citado por Gil y Morales, 2019, p. 162).

En tal sentido, la educación inclusiva ya no se limita a generar procesos educativos para las personas con una condición de educación especial, por una situación de discapacidad, por ejemplo, sino que amplía su mirada como una propuesta educativa para atender la heterogeneidad y diversidad del ser humano. Se empieza a reconocer que toda persona, independientemente de su capacidad, etnia, condición socioeconómica o funcionalidad, tiene derecho a ser comprendido en su singularidad. Es un eje que pretende romper con las prácticas tradicionales de las instituciones educativas, donde se daba atención únicamente a aquellas personas que en teoría presentaban alguna necesidad educativa, con énfasis en la deficiencia, donde se ignoran las diferentes habilidades que todo individuo tiene (Quesada, 2018, p. 49).

Por consiguiente, la atención a la diversidad es una forma de percibir y construir la realidad educativa, ya que implica otorgar el derecho a la educación que naturalmente le corresponde a cada persona, independientemente de las diferencias particulares que la caracterizan. Por lo tanto, 
se trata de aceptar que todo grupo social es diverso y que todas las personas tienen derecho a ser consideradas desde su individualidad y no desde un estándar homogéneo en el que difícilmente la mayoría podría encajar. Es entonces cuando se vuelve necesario entender que la igualdad está en reconocer la diferencia como un derecho y aprender a aprender de la diversidad, con el fin de eliminar la discriminación (Díaz, 2002, Lira y Ponce de León, 2006 y Parrilla, 2004, citados por Amaro, 2018).

En síntesis, en el pleno desarrollo del siglo XXI, se empieza a abordar la educación inclusiva para la diversidad como una propuesta educativa dirigida a reconocer la heterogeneidad y complejidad de la naturaleza del ser humano y la propia sociedad, desde diversos puntos de vista: ideológico, cultural, histórico, étnico, entre otros. Es así como se reconoce que la sociedad contemporánea tiene características interculturales, multiculturales y divergentes, que convierten a la educación en una propuesta compleja y retadora. Por tal motivo, los centros educativos no pueden ser vistos como instancias homogéneas, con una única y lineal propuesta educativa, sino que esta última requiere ser considerada y analizada desde sus múltiples factores que las definen y determinan.

\subsection{Educación a lo largo de la vida}

A diferencia del anterior eje, la educación a lo largo de la vida es una nueva propuesta de aprendizaje en el ser humano, la cual surge de los aportes de la UNESCO, con los informes de Faure (1972) y Delors (1996). Estos autores consideran que el aprendizaje no debe limitarse solo con el proceso de construcción de nuevos conocimientos, sino que debe ser guiado por un proceso de descubrimiento que conduce al bienestar personal y social, y que se produce con independencia de la edad o el entorno, tanto en la educación formal como en la no formal (UNESCO, 2014, citado por Salmeron, Escabajal y Martínez, 2016, p.320). Este eje considera el aprendizaje como una acción dialógica del ser humano, es decir, ser consciente y capaz de ejercitar un movimiento que permita saber qué hacer, por qué, cómo, cuándo y para qué se hacen las cosas, a partir del actuar en el contexto. Este proceso de pensamiento logra integrar nuevos aprendizajes significativos para la vida (Velasco, 2018).
La educación a lo largo de la vida reconoce cuatro principios fundamentales:

- Enseñar a vivir de manera conjunta, es decir, el diálogo permite la resolución de conflictos, en donde el proceso de socialización se basa en un enriquecimiento mutuo, debido al aporte beneficioso y complementario de diversos elementos.

- Enseñar a conocer, reforzando la capacidad de investigar de manera individual.

- Enseñar a hacer, aprender a través de la interacción y la acción, pero a través de la reflexión.

- Enseñar a ser: realizarse a ser uno mismo, tanto de manera individual, como colectiva, aumentando experiencias positivas de autoestima y afianzando la felicidad (Martínez y García, 2018, p. 4).

Otro elemento distintivo integrado en esta propuesta o eje educativo es que no se limita a un grupo etario o generación, sino que este proceso de aprendizaje es en cualquier estadio del desarrollo humano. La Declaración de Incheon (La Educación, F., 2015) reconoce que el aprendizaje es una condición que tiene el ser humano a lo largo de la vida, por lo que todas las generaciones deben tener la oportunidad de aprender y continuar aprendiendo, lo cual la convierte en un derecho intrínseco. Con este argumento, aparecen nuevamente los aportes de Torres (2005) y Velasco (2018), quienes consideran que este eje se encuentra inmerso en dos dimensiones: por un lado, la propuesta de aprender a lo largo de la vida (nacimiento hasta la muerte) y aprender a lo ancho de la vida (en todos los espacios de desarrollo del ser humano).

Ahora bien, al conocer la manera como se posiciona la educación como un derecho, desde el eje de inclusión para la diversidad y a lo largo de la vida, es posible cuestionar cuáles son las principales discusiones y preocupaciones de estos tres temas, en el contexto latinoamericano. El siguiente apartado pretende dar respuesta a esta pregunta a partir del análisis de los textos encontrados de autores del continente. 


\section{Las principales discusiones y preocupaciones latinoamericanas sobre los temas de estudio}

El análisis de la educación como un derecho, a partir de las políticas educativas impulsadas por los Estados, principalmente de corte neoliberal, es un tema de discusión en los artículos científicos de investigadores del continente latinoamericano, cuyo hallazgo se delimitó en dos principales discusiones. Estas cuestiones son, por un lado, cómo las políticas educativas fomentan la exclusión más que inclusión del ser humano, y por otro, la percepción de grupos y movimientos sociales latinoamericanos sobre el sentido de la educación en el continente.

Con respecto a la primera preocupación, se encontraron los aportes de Bello y Aguilar (2019), quienes manifiestan que las políticas educativas, especialmente relacionadas con el tema de la inclusión, se han enfocado en atender la parte individual, es decir, intervenir o actuar con aquellas personas que tienen un déficit, con el fin de desarrollar los diferentes atributos de las personas para que se integren en la sociedad. Estos autores consideran que esa visión de las políticas está enmarcada dentro de un paradigma simplista, basado en el pensamiento único en el marco de los límites o las fronteras disciplinares. Por eso, consideran la necesidad de cambiar las políticas educativas desde un paradigma de la complejidad, es decir, que la concepción del mundo rompe la continuidad, en una perspectiva holística, donde se incluya miradas diferentes. Por su parte, los colectivos sociales latinoamericanos, tales como comunidades indígenas, grupos y movimientos sociales que protegen y velan por los derechos de las personas con discapacidad, diversidad sexual, género, entre otros consideran que la educación como derecho implica una acción reflexiva y crítica ante las relaciones sociales hegemónicas, donde su fin es cambiar las situaciones que generan desigualdades y exclusiones (Mardones, 2012). y funcional del saber, al olvidar que la educación es un eje que permite construir el sentido del ser y la identidad del ser humano en la sociedad. Por eso, reconoce el esfuerzo de los pueblos indígenas, por rescatar nuevamente el sentido de la educación desde enfoques de desarrollo alternativos latinoamericanos, tales como el 'Buen Vivir' o Sumak Kawsay (Ecuador) o ‘Vivir Bien’ o Sumaq Qamaña (Bolivia). Por consiguiente, estos pueblos fomentan un tipo de educación intercultural crítica, al retomar los saberes de los pueblos originarios para contrarrestar las tendencias de homogenización, invisibilización y negación de la dignidad divergente del ser humano con su entorno.

\section{Los principales retos y desafíos para aplicar una educación inclusiva para la diversidad y a lo largo de la vida}

Ante la pregunta sobre cuáles son los principales retos y desafíos que tiene actualmente el contexto contemporáneo para aplicar una educación inclusiva para la diversidad y a lo largo de la vida, se identificaron los siguientes retos y desafíos:

\subsection{Revisar el espíritu y la lógica de las políticas o normativas nacionales e institucionales}

Investigadores sobre el tema de la educación inclusiva reconocen el avance normativo jurídico, pero evidencian grandes vacíos e inconsistencias en la manera de reconocer la educación como derecho (Zurita, 2011, Ruíz, 2012, Crespo, 2018, Castillo, 2019 y Ruiz, 2020). Por lo general, se tiende a fomentar una educación especial, segregada e integradora. Por eso se considera como uno de los principales retos revisar el espíritu y contenido de las normativas nacionales, regionales, locales e institucionales sobre la educación, las cuales no favorezcan la concepción de déficit o deficiencia, sino que fomenten la inclusión para la diversidad y a lo largo de la vida.
Uno de los autores que discute sobre esta preocupación en el continente es Crespo (2018), quien expone cómo en las últimas décadas, la óptica mercantilista ha impulsado políticas educativas con una visión reducida de la educación, la cual se limita a integrar solo la parte técnica 


\subsection{Generar acciones de sensibilización hacia la tolerancia y respeto a la diversidad}

A pesar del avance de la sociedad moderna, se observa un deterioro y retroceso de ciertos valores y principios sociales, entre ellos el respeto a la diversidad y la tolerancia. Día con día, en la vida cotidiana las personas enfrentan diversas manifestaciones de violencia, maltrato y desinterés por el reconocimiento y bienestar del "otro". Se fomenta más una vida individualizada, egoísta, competitiva, donde se impone el interés privado y propio, más que el colectivo. Además, se ve como amenazador "al otro", esas personas o sujetos que tienen condiciones diferentes de vida. Por eso, para fomentar el eje de la educación como derecho, desde la inclusión para la diversidad y a lo largo de la vida, se debe impulsar un fuerte y continúo proceso de sensibilización al respecto, a la tolerancia y a la comunicación asertiva. Estos procesos deben ser promovidos principalmente desde los agentes socializadores de la colectividad: familia, centros educativos, el ámbito laboral, espacios comunitarios, locales, entre otros.

\subsection{Fomentar experiencias de aprendizaje para todos}

Este reto implica abrir o ampliar la oferta educativa, ya sea formal e informal, a toda persona sin importar la condición etaria, de género, cultura, geográfica, entre otras. Implica ampliar la propuesta curricular, no centrarse en un currículo para el trabajo o el mercado laboral o desarrollo científico, sino también en generar experiencias educativas y de aprendizaje para todas las generaciones y con diversas temáticas para la vida. Un ejemplo de ello se percibe en propuestas educativas para el manejo de emociones, relaciones interpersonales, entre otros.

\subsection{Generar comunidades de aprendizaje, a partir la articulación de varios actores y sectores de la sociedad}

Otro importante reto es que el derecho de la educación no es solo una responsabilidad del Estado, sino de toda la sociedad, por lo que se percibe la necesidad de fomentar una red de alianzas para la protección, defensa y exigencia de la educación como derecho. Para Torres (2005), uno de los retos es fomentar comunidades de aprendizaje, al respecto menciona:

Es indispensable volver a vincular escuela, familia y comunidad, en una relación multidimensional y compleja, no de una sola vía (qué pueden hacer los padres de familia o la comunidad por la escuela), sino de doble vía (qué puede hacer la escuela por los padres de familia y por la comunidad y a la inversa) y conjuntamente (qué pueden hacer todos ellos juntos para asegurar la educación para todos y el desarrollo humano). Es aquí donde resulta útil pensar en un concepto articulador y con un fuerte potencial transformador como el de Comunidad de Aprendizaje (p. 84).

\subsection{Fomentar docentes con aptitudes y actitudes para trabajar la educación inclusiva para la diversidad y a lo largo de la vida}

Como un último reto y desafío, se encuentra la formación continua del personal docente para promover una educación inclusiva para la diversidad y a lo largo de la vida. Esta formación no solo debe ir dirigida en la preparación de conocimientos, habilidades y destrezas profesionales, sino también hacia un fomento de la experiencia y estilo de vida (aptitudes y actitudes) a favor del nuevo paradigma educativo.

\section{Conclusiones generales}

A manera de conclusiones, se puede decir que el derecho a la educación se reconoce y legitima a finales del siglo XIX y el inicio del siglo XX, por reconocer un grupo de derechos humanos, que son irrenunciables y fundamentales. Este derecho parte del Pensamiento Republicano, que busca fomentar prácticas democráticas e igualitarias entre los seres humanos y las comunidades, es decir, un estilo de vida cívico, como garante para alcanzar los principios de justicia, paz, armonía, comprensión y tolerancia. 
El fin último de la educación, desde las normativas internacionales, es el de fomentar el desarrollo integral del ser humano en la sociedad y a la vez, fortalecer el disfrute y alcance de otros derechos y libertades fundamentales. Por otro lado, se establece que la sociedad, mediante el papel del Estado, debe garantizar todas las condiciones para la educación de todas las personas, de manera universal, obligatoria y gratuita. Con la intención y esfuerzo de expandir e integrar el derecho a la educación en todos los sectores y grupos poblacionales, se manifiestan dos fenómenos en los últimos veinte años:

a) La ampliación de la cobertura de los servicios educativos, que ha evidenciado situaciones de disparidad y desigualdad, los cuales se convierten en elementos violadores y discriminatorios de este derecho, porque existe un interés por integrar al ser humano al sistema educativo, desde la visión homogénea, estandarizada y única, que no se adecua a todas las diferentes manifestaciones del ser humano y la sociedad.

b) Las políticas educativas de corte neoliberal, que surgen a inicio del XXI, tiene otro rumbo diferente, que afecta el espíritu de fomentar la educación como derecho, pues tienen el matiz de procurar lineamientos políticos compensatorios, asistenciales y focalizadores, especialmente para las poblaciones en condición de pobreza o desventaja social.

Ante estos dos fenómenos, uno de los principales retos y desafíos de la educación en el presente siglo XXI es recuperar su sentido como un derecho, este fin tiene el Marco de Acción de la Educación hacia el 2030, el cual incorpora dos ejes que resultan tener una visión novedosa en el alcance y práctica de la educación como derecho: la educación inclusiva para la diversidad y a lo lago de la vida.

La educación inclusiva también ha evolucionado por varios enfoques y paradigmas en la actuación de lo diverso. Inicia con una visión de lo diverso como deficiencia o déficit, lo cual implica una educación especial e integradora, para culminar con una visión más inclusiva, donde en la actualidad tiene una tendencia a reconocer las diversas manifestaciones del ser humano en la sociedad. Es así que esta propuesta educativa es un espacio de reconocimiento y tolerancia a las diferencias entre los grupos poblacionales, por su condición de género, raza, edad, identidades sexuales, identidades culturales, condiciones físicas, entre otras. Se promueve una educación dirigida hacia el reconocimiento de la heterogeneidad y complejidad de la naturaleza del ser humano, desde diversos puntos de vista: ideológico, cultural, histórico, étnico, entre otros. Es así como se reconoce que la sociedad contemporánea tiene características interculturales y multiculturales.

Con respecto al eje "educación a lo largo de la vida", es considerado como una nueva propuesta de aprendizaje, que surge del diálogo y la interacción que tiene el ser humano con su contexto. Implica un movimiento que permite saber qué hacer, por qué, cómo, cuándo y para qué se hacen las cosas, a partir de la interacción en la sociedad. Este tipo de aprendizaje se manifiesta en cualquier etapa de desarrollo (nacimiento hasta la muerte) y desde la esfera formal y no formal.

La educación debe ser una propuesta más reflexiva y crítica ante las relaciones sociales hegemónicas y las contradicciones reproducidas por el sistema capitalista, con el modelo de desarrollo neoliberal. Es por eso que dentro del continente latinoamericano se manifiestan dos principales preocupaciones y discusiones alrededor de la educación como derecho. Por un lado, el análisis de cómo las políticas educativas fomentan la exclusión; por otro lado, la pérdida del sentido de la educación como un derecho.

Este artículo presenta retos y desafíos para aplicar la educación como derecho, desde los dos ejes de interés de análisis. Estos retos y desafíos son revisar el espíritu y la lógica de las políticas y normativas nacionales e institucionales; generar acciones de sensibilización hacia el respeto a la diversidad; fomentar experiencias de aprendizaje para todo tipo de personas; generar comunidades de aprendizaje, a partir la articulación de varios actores y sectores de la sociedad y fomentar docentes con aptitudes y actitudes para trabajar la educación inclusiva para la diversidad y a lo largo de la vida. 


\section{Bibliografía}

Amaro, M. (2019). "Formación docente para la atención a la diversidad en Educación Superior: una búsqueda de posibilidades para la enseñanza”. Revista de Educación Inclusiva, 12(1), 51-66. Disponible en: https://dialnet.unirioja.es/servlet/ articulo?codigo $=7017824$

Bello, J. y Aguilar, M. (2019). "Educación inclusiva en Latinoamérica”. Brazilian Journal of Latin American Studies, 18(35), 55-76. Doi: 10.116o6/ issn.1676-6288.pJuanrolam.2019.164124

Beltrán, Y., Martínez, Y. y Vargas, Á. (2015). El sistema educativo colombiano en el camino hacia la inclusión: Avances y retos. Educación y Educadores, 18(1), 62-75. Doi: 10.5294/ edu.2015.18.1.4

Calderón, P. (2015). El derecho humano a la educación como camino hacia la democracia y hacia el fin de la guerra. Diálogos De Saberes, (43), 73-89. Disponible en: https://doi.org/10.18041/01240021/dialogos.43.163

Castillo, N. (2019). "Educación Inclusiva: Contradicciones, debates y resistencias". Praxis Educativa, 23(3), 4. Doi: https://dx.doi.org/10.19137/ praxiseducativa-2019-230304

Crespo, C. (2018). "Semillas y caminos para la educación latinoamericana en tiempos de incertidumbre". Revista Educação e Emancipação, 11(3), 3652. Doi: http://dx.doi.org/10.18764/2358-4319. v11n3p36-52

Gil, J. y Morales, M. (2019). "Diversidad y educación inclusiva en las universidades: cambiar estigmas y ordenar conceptos”. Revista Metropolitana de Ciencias Aplicadas, 2(1), 160-165. Disponible en: http://remca.umet. edu.ec/index.php/REMCA
Gluz, N., Rodrigues, C. y Oliveira, D. (2018). "Políticas de inclusión y extensión de la obligatoriedad escolar: alcances, deudas y desafíos en la materialización del derecho a la educación”. Archivos Analíticos de Políticas Educativas, 26(155). Disponible en: http://dx.doi.org/10.14507/epaa.26.3788

La Educación, F. (2015). Declaración de Incheon. Educación 2030: Hacia una educación inclusiva y equitativa de calidad y un aprendizaje a lo largo de la vida para todos. ED/WEF2015/MD/3. Disponible en: http://unesdoc. unesco. org/ images/o023/002331/233137s. pdf.

Mardones, R. (2012). "Movimientos sociales, derechos humanos y educación: Hacia una visión compleja desde el pensamiento de Emmanuel Lévinas”. Revista Electrónica de Psicología Política, 9 (27), 96-111. Disponible en: http:// web.a.ebscohost.com/ehost/pdfviewer/ pdfviewer?vid=1\&sid=379ac679-6d66-463686ef-675333227f4c\%40sessionmgr40o6

Martínez, N. y García, A. (2018). Educación intergeneracional: un nuevo reto para la formación del profesorado. REXE-Revista de Estudios y Experiencias en Educación, 17(33), 113-124. Doi: https://doi.org/10.21703/ rexe.20181733nmartinez7

Naciones Unidas, (1999). «Cuestiones sustantivas que se plantean en la aplicación del Pacto Internacional de Derechos Económicos, Sociales y Culturales: Observación General 12». Consejo Económico y Social de Naciones Unidas, $20^{\circ}$ período de sesiones, Ginebra, Suiza, 26.

Naciones Unidas (2001). Observación General No. 1 Párrafo 1 del artículo 29: propósitos de la Educación. 17 de abril de 2001. CRC/GC/2001/1 Naciones Unidas. Disponible en: http://tbinternet. ohchr. org/_layouts/treatybodyexternal/Download. aspx. 
Quesada, L. (2018). "Atención a la diversidad en la Educación Superior: Una propuesta desde el Trabajo Comunal Universitario (TCU)". Wimb Lu, 13(1), 47-61. Doi: 10.15517/WL.V13I1.33225

Ruiz, M. (2012). "Derecho a la educación: política y configuración discursiva”. Revista Mexicana de Investigación Educativa, 17(52), 39-64.Disponible en: http://www.scielo.org.mx/pdf/rmie/v17n52/ v17n52a3.pdf

Ruiz, G. (2020). "El derecho a la educación y su desarrollo conceptual desde una perspectiva histórica". Cadernos de História da Educação, 19(2), 54456o. Disponible en: https://doi.org/10.14393/ che-v19n2-2020-16

Salmerón, J., Escarbajal, A. y Martínez, S. (2018). “Estudio sobre una experiencia educativa con mujeres mayores en centros sociales. Implicaciones para el aprendizaje a lo largo de la vida". Revista Complutense de Educación, 29(2), 317. Doi: http:// dx.doi.org/10.5209/RCED.52381

Torres, R. (2005). Justicia educativa y justicia económica. 12 tesis para el cambio educativo. Estudio encargado por el Movimiento Internacional de Educación Popular y Promoción Social. Disponible en: https://ialnet.unirioja.es/servlet/ articulo?codigo $=1351881$

Valesco, (2018). "Aprender en el aprender a lo largo de la vida”. Revista Iberoamericana de Educación, 76(1), 125-144. Doi: https://doi.org/10.35362/ rie 7612648

Zurita, Ú. (2011). "Los desafíos del derecho a la educación en México a propósito de la participación social y la violencia escolar". Revista Mexicana de Investigación Educativa, 16(48), 131-158. Disponible en: https://www.redalyc.org/articulo. oa?id=140/14015561007 